\title{
Sentinel node in melanoma patients: triple negativity with routine techniques and PCR as positive prognostic factor for survival
}

\author{
Valeria C Denninghoff ${ }^{1}$, Jorge Falco², Andrea G Kahn, Víctor Trouchot ${ }^{2}$, \\ Hector P Curutchet ${ }^{2}$ and Boris Elsner ${ }^{3}$
}

${ }^{1}$ Department of Pathology, Center for Medical Education and Clinical Investigation, Buenos Aires, Argentina; ${ }^{2}$ Division of Surgical Oncology, Hospital de Clínicas 'José de San Martín', University of Buenos Aires School of Medicine, Buenos Aires, Argentina and ${ }^{3}$ Department of Pathology, Hospital de Clínicas 'José de San Martin', University of Buenos Aires School of Medicine, Buenos Aires, Argentina

\begin{abstract}
Lymph node mapping and sentinel lymph node biopsy are currently used to stage patients with cutaneous malignant melanoma. Immunohistochemical stains contribute to the detection of micrometastases; however, molecular biology techniques are associated with better diagnostic sensitivity. Sixty sentinel lymph nodes were included in this study. The primary lesions were malignant melanoma stage I or II, with a follow-up of longer than 2 years. Sentinel lymph nodes were studied with hematoxylin-eosin, immunohistochemistry for S-100 and HMB-45, and molecular biology techniques (reverse transcription (RT)-PCR) for the detection of tyrosinase messenger RNA. In 15 of 60 cases (25\%), tyrosinase was detected by RT-PCR; three of these cases were also positive by immunohistochemistry. The population was divided into three groups: (i) hematoxylin-eosin-I immunohistochemistry +/molecular biology techniques + (3 cases); (ii) hematoxylin-eosin-/immunohistochemistry-/molecular biology techniques + (12 cases); (iii) hematoxylin-eosin-/immunohistochemistry-/ molecular biology techniques - (45 cases). Correlation of the groups with overall survival showed the following: (i) 2 of 3 patients died (67\%); (ii) 5 of 12 died (42\%), and (iii) all 45 patients are alive, with no lymphadenectomy and a median follow-up of 84 months. The inclusion of molecular biology techniques appears to be of great value for the detection of sentinel lymph node micrometastases in patients with cutaneous malignant melanoma. In our series, those patients who showed negativity with all the three methods had a null recurrence rate. Therefore, this triple negativity could be a positive prognostic factor for overall survival. Our findings suggest the possibility of molecular oncological staging, which would allow the selection of patients with submicroscopic metastases for a complete treatment.
\end{abstract}

Modern Pathology (2008) 21, 438-444; doi:10.1038/modpathol.3801020; published online 25 January 2008

Keywords: malignant melanoma; sentinel lymph node; PCR; tyrosinase; positive prognostic factor; overall survival

Malignant melanoma is the most aggressive tumor of the skin. ${ }^{1}$ This malignant neoplasm only represents $4 \%$ of skin tumors, accounting for $2 / 3$ deaths. ${ }^{2}$ Over the last years, there has been growing concern about malignant melanoma. There is a high incidence of this condition in several countries. Between 1997 and 2001, malignant melanoma mortality rate in Argentina was 1.2 in 100000 men per year, and 0.8 in 100000 women per year, which accounts for 354

Correspondence: Dr VC Denninghoff, PhD, Department of Pathology, Center for Medical Education and Clinical Investigation, Arcos $18531^{\circ} \mathrm{B}$, Buenos Aires C1428AFA, Argentina.

E-mail: vdenninghoff@cemic.edu.ar

Received 26 July 2007; revised 2 December 2007; accepted 3 December 2007; published online 25 January 2008 deaths per year. ${ }^{3}$ Between 85 and $90 \%$ of malignant melanoma patients present at early stages, when cure is still possible. Survival at 5 years of patients with recently diagnosed localized primary melanoma (stages I and II, as designated by the American Joint Committee on Cancer (AJCC)) is about $80 \%$, compared with a $35 \%$ survival in case lymph node metastases occur (AJCC stage III). ${ }^{4}$ In these cases, conventional treatment of patients with lymph node metastases includes primary tumor removal and lymph node dissection or lymphadenectomy. ${ }^{5,6}$ Lymph node status in patients with malignant cutaneous melanoma is the most important prognostic factor for disease outcome, with a $50 \%$ decrease in the 5-year survival rate when subclinical metastases are detected at stages I and II. ${ }^{7}$ 
Selective lymphadenectomy is a relatively new procedure that consists of the intraoperative identification of the first node in the nodal basin, the sentinel lymph node, which is removed for pathological examination. ${ }^{8}$ To identify sentinel lymph node, all patients need to undergo preoperative lymphoscintigraphy and a vital dye perilesional injection, followed by $\gamma$-probe-guided surgery. The procedure is minimally invasive, safe, and inexpensive, and allows the selection of patients who could benefit from elective node dissection. ${ }^{9}$ This strategy contributed to a wider knowledge, staging, diagnosis, and treatment of this condition. ${ }^{10}$

It has been demonstrated that methods such as hematoxylin-eosin and immunohistochemistry, with a combination of markers such as S-100 and HMB-45 and/or MART-1, fail to detect some tumor cells, due to their sensitivity. Therefore, methods for the detection of micrometastases should be optimized to reduce the rate of false-negative sentinel lymph node readings. Molecular biology techniques are sensitive and proved to be able to detect a larger number of cases with possible metastatic deposits. ${ }^{11,12}$ PCR is a molecular biology technique that identifies one neoplastic cell in one million normal cells, and it could be used as an additional method for the detection of malignant nodal metastatic melanomas in the sentinel lymph node. The messenger RNA (mRNA) coding for tyrosinase was used as the target sequence for reverse transcription (RT). Tyrosinase gene maps in the 11q14q21 chromosomal region, and the $60-$ to $70-\mathrm{kDa}$ protein translated is essential for melanin synthesis. Since tyrosinase is a key enzyme during melanin synthesis, the mRNA for this gene will only be expressed in cells actively producing the pigment. Thus, the presence of tyrosinase mRNA in a lymph node could be associated with micrometastases. ${ }^{13,14}$ The importance of the identification and evaluation of the sentinel lymph node in patients with malignant melanoma stages I and II, especially with Breslow thickness $1 \mathrm{~mm}$ or more, has been extensively demonstrated in multiple studies worldwide during the last decade. Knowledge of lymph node status in these patients is essential to accurately determine its stage, to assess prognosis (nodes are the most important prognostic factor), and to select the best treatment options. The sixth edition of the AJCC Cancer Staging manual recognizes sentinel lymph node dissection and indicates that occult metastases (micrometastases) should be reported as nodal involvement (modifier ' $a$ ' in N1/2). The College of American Pathologists protocol based on the AJCC staging manual recommends that identification of isolated tumor deposits $(<0.2 \mathrm{~mm})$ by morphologic or nonmorphologic methods (including hematoxylin-eosin, immunohistochemistry, and molecular biology techniques) should be denoted in the report, with no change in the nodal status (No). ${ }^{15}$
This study was carried out to assess the sensitivity of tyrosinase mRNA amplification by RT-PCR in sentinel lymph node, and to correlate these results with the clinical outcome of patients at the Hospital de Clínicas.

\section{Materials and methods}

The study population was selected from 598 patients with malignant melanoma who underwent treatment at the Hospital de Clínicas, Buenos Aires, from 1994 to 2004. Only 364 patients had stage I and II (AJCC) melanoma, and 123 underwent lymphatic mapping and sentinel lymph node biopsy. ${ }^{16}$ Of the total sample, 60 sentinel lymph nodes, negative on routine histology, were selected for the present study because they included a cryopreserved portion and a follow-up of longer than 2 years. The median follow-up of the 60 patients was 74 months, with a range of 9-103 months. All the sentinel lymph nodes were bisected; a central slice $(20 \%$ of sentinel lymph node more than $0.5 \mathrm{~cm}$ diameter) was frozen $\left(-70^{\circ} \mathrm{C}\right)$ for molecular analysis, and the remaining tissue was formalin-fixed and paraffinembedded. They were preoperatively (lymphoscintigraphy) and intraoperatively identified, further removed, and histopathologically studied. Serial sections were obtained for hematoxylin-eosin and immunostaining for S-100 protein and HMB-45, whereas molecular biology techniques were used to determine the presence of micrometastases. ${ }^{13,17}$ Median age was 54 years, with a range of 23-84 years. Twenty-seven patients were females (Table 1). The primary melanoma was located in the chest in 24 patients, the lower limb in 14, the arm in 12, the head and neck in 9, and 1 in the vulva. Thirty-nine patients presented with a superficially spreading melanoma, 17 with a nodular melanoma, 3 with an acral lentiginous melanoma, and 1 with a desmoplastic melanoma.

Serial sections $(4 \mu \mathrm{m})$ were obtained from formalin-fixed and paraffin-embedded sentinel lymph node portions, and were immunostained for S-100 protein (Biogenex, San Ramon, CA, USA, 1:400) and HMB-45 (Dako, Carpinteria, CA, USA, 1:50). These sections were incubated with the primary serum. The avidin-biotin method was carried out with the Vectastain Elite kit (Vector Labs, Burlingame, CA, USA); diaminobenzidine was used as a chromogen and hematoxylin was used as a counterstain.

Total cellular RNA (tRNA) was extracted from fresh-frozen sentinel lymph node portions using SV Total RNA Isolation System (Promega Corporation, Madison, WI, USA). The purity and yield of tRNA were measured by spectrophotometry, and 1-2 $\mu \mathrm{g}$ of tRNA was used for RT. Better results were obtained after heating the tRNA with the specific outer reverse primer for $10 \mathrm{~min}$ at $90^{\circ} \mathrm{C}$ (hot start). Complementary DNA (cDNA) was obtained after 
Table 1 Characteristics of tumors and median follow-up in the different groups

\begin{tabular}{lccc}
\hline Group & $I$ & $I I$ & $I I I$ \\
\hline Hematoxylin-eosin & - & - & - \\
Immunohistochemistry & + & + & - \\
RT-PCR & + & $12(20)$ & $45(75)$ \\
Number of cases (\%) & $3(5)$ & $45(26-62)$ & $90(23-84)$ \\
Median age in years (range) & $46(31-67)$ & $39(24-93)$ & $22 / 3$ \\
Sex (male/female) & $2 / 1$ & $1.5(1.2-8.5)$ & $84(24-103)$ \\
Median FU in months (range) & $35(9-72)$ & III:4 (33) & IV:4 (33) \\
Median Breslow index in mm (range) & $1.6(1.5-4.1)$ & V:4 (33) & II:11 (31) \\
Clark's level-N (\%) & IV:3 (100) & DOD:5/12 & IV:11 (31) \\
& & SL-w:5/12 & V:3 (9) \\
& & SL-wo:2/12 & DOD:0/45 \\
Follow-up data/number of cases (\%) & DOD:2/3 & SL-w:0/45 \\
& SL-w:1/3 & SL-wo:0/3 & SL-wo:45/45
\end{tabular}

RT, reverse transcriptase; DOD, died of the disease; SL-w, survived with lymphadenectomy; SL-wo, survived without lymphadenectomy.

adding $25 \mu \mathrm{l}$ reaction mixture containing $200 \mathrm{U}$ of Moloney Murine Leukemia Virus Reverse Transcriptase (M-MLV RT), (Promega Corporation), $50 \mathrm{mM}$ Tris-HCl (pH 8.3), $75 \mathrm{mM} \mathrm{KCl,} 3 \mathrm{mM} \mathrm{MgCl}_{2}, 75 \mathrm{pmol}$ of specific outer antisense primer, $200 \mu \mathrm{M}$ of each dNTP, and $40 \mathrm{U}$ of Recombinant RNasin $\AA$ Ribonuclease Inhibitor (Promega Corporation) to the previous tube. Complementary DNA synthesis was performed at $42^{\circ} \mathrm{C}$ for $1 \mathrm{~h}$, and was directly used for PCR amplification or stored at $-20^{\circ} \mathrm{C}$. Tyrosinase cDNA was amplified and reamplified (double-round PCR) using specific outer and nested primers. ${ }^{11}$ For the first PCR, we added $20 \mu \mathrm{l}$ of the PCR mixture containing a final concentration of $10 \mathrm{mM}$ Tris-HCl (pH 9.0), $50 \mathrm{mM} \mathrm{KCl}, 2.5 \mathrm{mM} \mathrm{MgCl}_{2}, 75 \mathrm{pmol}$ of specific outer sense primer, and $1 \mathrm{U}$ of Taq DNA Polymerase (Promega Corporation) to the cDNA. PCR was performed in a Triothermoblock cycler (Biometra, Göttingen, Germany) under the following conditions: $3 \mathrm{~min}$ at $95^{\circ} \mathrm{C}, 60 \mathrm{~s}$ at $95^{\circ} \mathrm{C}, 60 \mathrm{~s}$ at $62^{\circ} \mathrm{C}$ for 30 cycles, and a final 5 -min extension at $72^{\circ} \mathrm{C}$. For reamplification, $5 \mu \mathrm{l}$ of the first PCR were used in a second round of 30 cycles with nested primers under the same conditions. Oligonucleotide sequence of outer primers for human tyrosinase was HTYR-1 (5'-TTGGCAGATTGTCTGTAGCC-3') and HTYR-2 (5'-AGGCATTGTGCATGCTGCTT-3'), which amplifies a product of $284 \mathrm{bp}$; nested primers were HTYR-3 (5'-GTCTTTATGCAATGGAACGC- $\left.3^{\prime}\right)$ and HTYR-4 (5'-GCTATCCCAGTAAGTGGACT-3'), which amplify a nested PCR product of $207 \mathrm{bp}$. RNA integrity was verified by the amplification of glyceraldehyde-3-phosphate dehydrogenase, with identical reaction mixtures and incubation programs, except for the primers that produce a 315-bp product using outer primers GAPD-1 (5'-GACAACAGCCTCAAG ATCATC- $\left.3^{\prime}\right)$ and GAPD-2 (5'-GACGGCAGGTCAG GTCCACCA-3'), and a 248-bp nested product with nested primers GAPD-3 (5'-AATGCCTCCTGCAC CACC- $\left.3^{\prime}\right)$ and GAPD-4 (5'-ATGCCAGTGAGCTTC
CCG-3'). ${ }^{11}$ Since glyceraldehyde-3-phosphate dehydrogenase is a key enzyme during glycolysis, its mRNA will be expressed in every cell. Thus, it becomes an adequate RNA purification control, which indicates the presence of intact RNA liable to amplification. PCR products were analyzed by electrophoresis on a $9 \%$ polyacrylamide gel, followed by ethidium bromide staining, and were further photographed. A 100-bp ladder (Promega Corporation) was used as a size reference marker. Different positive controls were included in this study: a malignant melanoma biopsy of the skin, a lymph node with massive metastatic malignant melanoma, seven sentinel lymph nodes with malignant melanoma metastases stage III (positive histology), and a sentinel lymph node with amelanotic malignant melanoma metastases stage III (positive histology). Two nerve biopsies were included as negative controls. Primer design can resolve the DNA presence. A positive result was only considered in the presence of the 207-bp nested band for human tyrosinase mRNA and the 248-bp nested band for glyceraldehyde-3-phosphate dehydrogenase. To verify tyrosinase-specific RT-PCR products, restriction fragment length polymorphism was performed, and the specific bands were purified from the polyacrylamide gel and were sequenced by the DYEnamic ET Terminator Cycle Sequencing kit and analyzed with the ABI PRISM ${ }^{\mathbb{R}}$ DNA Sequencing Analysis Software.

These results were correlated with age, sex, Breslow index, and Clark level. Differences in survival were determined. Descriptional data were analyzed with median, mean, and range in continuous variables. Categorical variables were expressed in rates or relative frequencies. Continuous variables were analyzed with the $t$-test or with a non-parametric test if data distribution was normal. Categorical variables were analyzed with the Fisher's exact test. 


\section{Results}

Few neoplastic cells with S-100 and HMB-45 were found in three patients in the subcapsular region or in the lymph node parenchyma. Tumor cells were positive in the nuclei and cytoplasm with S-100, and had a granular cytoplasmic immunostain with HMB-45. Of the 60 patients examined through molecular biology techniques, 15 were positive $(25 \%)$. In 12 of $60(20 \%)$ samples, only the nested RT-PCR was positive. Clear signals were seen at $207 \mathrm{bp}$ for tyrosinase mRNA and at $248 \mathrm{bp}$ for glyceraldehyde-3-phosphate dehydrogenase mRNA. Control results were as expected. Tyrosinase mRNA sequence was according to NCBI reference sequences: NM000372.2. Restriction fragment length polymorphism results were as expected: the 207-bp tyrosinase mRNA RT-PCR nested fragment digested with $\mathrm{NCOI}$ (c/catgg) determined 135 and 72 bp bands. In a previous study, we analyzed, by molecular biology techniques, the tyrosinase expression in paraffin-embedded sections from the same sentinel lymph nodes. No comparative results were obtained between both types of tissue conservations, and the sensitivity is lower in paraffin-embedded than in fresh-frozen tissues.

The population was divided into three groups: in the hematoxylin-eosin-/immunohistochemistry + / molecular biology techniques + group, 2/3 (67\%) patients died at 9 and 35 months, and the other patient is alive, with a 72-month follow-up, and has undergone therapeutic lymphadenectomy. In the second group, hematoxylin-eosin-/immunohistochemistry-/molecular biology techniques,$+ 5 / 12(42 \%)$ died at $33,36,40,45$, and 48 months, and the other patients are alive. Five of them have undergone lymphadenectomy, with a median follow-up of 36 months, with a range of 2470 months. The other two have not undergone lymphadenectomy, with a follow-up of 84 and 93 months. In the third group, hematoxylin-eosin-/ immunohistochemistry-/molecular biology techniques-, all $45(100 \%)$ patients are alive, with a median follow-up of 84 months, and a range of 24-103 months. They have not undergone lymphadenectomy, and 4/45 patients have local recurrence.

The statistical values for immunohistochemistry and molecular biology techniques were calculated in patients without lymphadenectomy, using death from the disease as a gold standard. Immunohistochemistry values were sensitivity: 0.29 ; specificity: 1.00; false-positive rate: 0.00 ; false-negative rate: 0.71 ; positive predictive value: 1.00 ; and negative predictive value: 0.90 . Values of molecular biology techniques were sensitivity: 1.00; specificity: 0.96; false-positive rate: 0.22 ; false-negative rate: 0.00 ; positive predictive value: 0.78 ; negative predictive value: 1.00; and positive likelihood ratio: 25 . The likelihood ratio comprises both the sensitivity and specificity of the test, and provides a direct estimate of how a test result will change the odds of having a disease. The 'positive likelihood ratio' shows how to increase the probability of disease in case of a positive test. Positive likelihood ratio of more than 10 indicates a large and often-conclusive increase in the likelihood of disease. Patients' overall survival without lymphadenectomy at 60 months was $0 \%$ in group I (hematoxylin-eosin-/immunohistochemistry + /molecular biology techniques + ); $29 \%$ in group II (hematoxylin-eosin-/immunohistochemistry-/molecular biology techniques + ); and $100 \%$ in group III (hematoxylin-eosin-/immunohistochemistry-/molecular biology techniques-), as shown in the survival curve (Figure 1). According to the follow-up of results, the population was divided into three groups as follows: group A, patients who died of the disease; group B, patients who survived with lymphadenectomy; and group $\mathrm{C}$, patients who survived without lymphadenectomy. The negative results of molecular biology techniques determined a decreasing number of patients who died of the disease, but an increasing number of diseasefree survival patients as well. This population division was confirmed in patients who survived without lymphadenectomy, with a Fisher's exact value $=0.000$.

\section{Discussion}

There is a high incidence of malignant melanoma in the population. This is the first sentinel lymph node survival study with immunohistochemistry and molecular biology techniques in an Argentinean population with malignant melanoma stages I and II. Pathologic stage I melanoma and stage II melanoma affect those patients with no evidence of regional or distant metastases, given the absence of nodal metastases after careful examination of regional lymph nodes, and the absence of distant metastases after routine clinical and radiological examination. Pathologic stage III melanoma patients present with regional metastases, either in regional lymph nodes or at intralymphatic sites. The quantitative

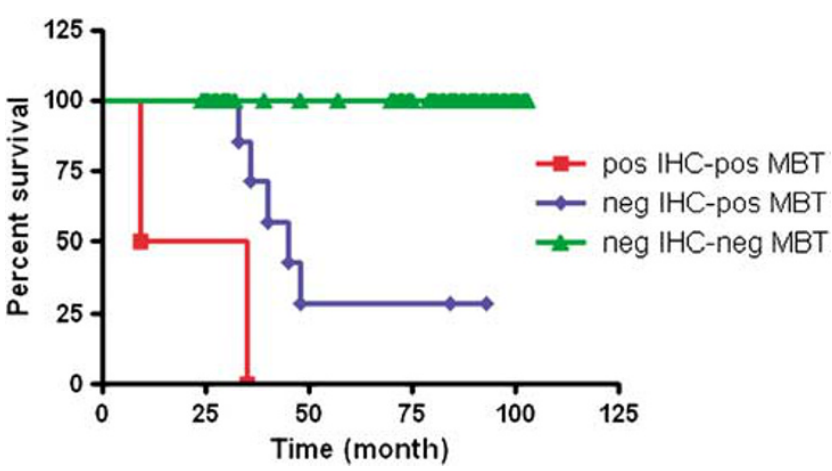

Figure 1 Fifty-four patients' overall survival without lymphadenectomy according to time of follow-up. Log-rank test: $\chi^{2}$ (2 df):66,31 $P$-value < 0.0001. IHC, immunohistochemistry; MBT, molecular biology techniques; pos, positive; neg, negative. 
classification of pathologic nodal status requires careful examination of the surgically resected nodal basin by a pathologist, and a report on the actual number of lymph nodes examined and the number of nodal metastases identified. With the widespread use of sentinel node lymphadenectomy, there is considerable stage migration of patients previously staged as node negative, who, in fact, had undetected nodal metastases. These previously understaged stage III patients have revealed an extraordinary heterogeneity of metastatic risk for stage III melanoma. Thus, 10-year survival rates among various subgroups of pathologic stage III patients vary widely, ranging from 9 to $63 \% .{ }^{4}$ Fiveyear survival of patients with localized primary melanoma recently diagnosed (stages I and II, as designated by the AJCC) is about $80 \%$, compared with a $35 \%$ survival in case of lymph nodes (AJCC stage III). ${ }^{4}$

The most important melanoma predictor factor is regional lymph node tumor status. ${ }^{13,18}$ When lymph node metastases appear, the prognosis based on the primary tumor fails to predict recurrence or survival. As the detection of micrometastases is a prognostic factor with an impact on therapy (lymphadenectomy) and disease prognosis, micrometastases detection methods should be optimized to minimize false-negative results. ${ }^{19}$ Different opinions exist about regional lymph approach in malignant melanoma patients. ${ }^{20}$ Some authors prefer lymphadenectomy only in the presence of metastasis, whereas others prefer prophylactic lymphadenectomy even with an apparently normal lymph node in patients at a high risk for lymph node metastases. ${ }^{21}$ The latter suggests that melanoma dissemination occurs sequentially, first in regional nodes and then in distant regions. ${ }^{6,22}$

Approximately $10 \%$ of patients with negative sentinel lymph node examined with routine techniques (hematoxylin-eosin and immunohistochemistry) develop distant metastasis. To increase sensitivity in the detection of nodal metastases, molecular biology techniques have been introduced to ascertain the presence of mRNA linked to genes highly expressed in malignant melanoma. ${ }^{10,23}$ Tyrosinase, the key enzyme in melanin biosynthesis, is expressed both in normal melanocytes and in malignant melanoma cells. ${ }^{24}$ The molecular biology techniques performed have higher sensitivity and specificity than routine techniques (hematoxylineosin and immunohistochemistry). Moreover, they are useful for the detection of micrometastases in sentinel lymph node from malignant melanoma patients. Our primer design can resolve DNA presence. Some authors suggested that nested RT-PCR overdiagnoses occult metastasis in patients with malignant melanoma, thus leading to overtreatment. ${ }^{13}$ Since our negative control results were as expected, we considered that tyrosinase mRNA detection did not lead to false-positive results or overdiagnosis. In the presence of tyrosinase machinery, a positive RT-PCR is theoretically possible. Therefore, nodal nevi could lead to falsepositive results. The false-negative rate was a good indicator of error risk. The false-negative rate to immunohistochemistry was 71 and null for PCR.

On the other hand, we tested molecular biology techniques through other sources of possible falsenegative results: an amelanotic malignant melanoma is a form of malignant melanoma in which malignant cells do not produce melanin pigment. Amelanotic malignant melanoma may be pink, red, or have light brown, tan, or gray at the edges, and represents a clinic variant in $2 \%$ of patients with malignant melanoma. Amelanotic malignant melanoma survival does not differ from malignant melanoma. Amelanotic malignant melanoma has an unusual pigment synthesis, because melanin acidification keeps the pigment in the endoplasmic reticulum. Therefore, it can neither enter the Golgi complex nor participate in the L-tyrosine metabolism. ${ }^{25}$ Our study design also includes a metastasis sentinel lymph node from a patient with amelanotic malignant melanoma. We had no difficulty in detecting micrometastases in these sentinel lymph nodes, since tyrosinase mRNA was present in melanocytes, but was a non-functional protein. ${ }^{25}$

A nested technique for the detection of tyrosinase mRNA in sentinel lymph node is a good choice, provided that several measures are taken to avoid contamination and to prove both specificity and sensitivity. In the different series, the number of cases detected by tyrosinase RT-PCR (hematoxylineosin and immunohistochemistry negative sentinel lymph node) ranges from $13.5 \%$ to as high as $63 \%$, and recurrence rate from 10 to $25.6 \%$. In our series, $12 / 60(20 \%)$ cases were positive with RT-PCR alone, and recurrence/death rate was 5/12 (42\%). The sensitivity to detect micrometastases was $5 \%$ (3/60) higher in immunohistochemistry than in hematoxylin-eosin, and $20 \% \quad(12 / 60)$ higher in molecular biology techniques than in immunohistochemistry. The identification rate, which determines the possibility to find micrometastases with the method used, was 0.22 for immunohistochemistry and 0.78 for molecular biology techniques. Then, we considered that immunohistochemistry and molecular biology techniques were complementary. Although concerns have been raised regarding the destruction of the tissue to be submitted for solution-phase RT-PCR (the method widely used) and the lack of morphologic correlation, there seems to be a good association between the positivity by this method alone, and the overall survival of patients in the reported series. The overall survival reported in the literature in sentinel lymph node hematoxylin-eosin-/immunohistochemistry $+/$ molecular biology techniques + patients was $39 \%$, in sentinel lymph node hematoxylineosin-/immunohistochemistry-/molecular biology techniques + patients it was $82 \%$, and in patients with triple negativity it was $98 \%{ }^{23}$ In our study, the 
correlation of groups with overall survival (without lymphadenectomy) at 60 months was $0 / 2(0 \%)$ in group I (hematoxylin-eosin-/immunohistochemistry + /molecular biology techniques + ), 2/7 $(29 \%)$ in group II (hematoxylin-eosin-/immunohistochemistry-/molecular biology techniques + ), and all 45 patients (100\%) in group III (hematoxylin-eosin-/immunohistochemistry-/molecular biology techniques-). Four patients in the last group had local recurrence.

The molecular biology techniques performed in sentinel lymph nodes can only evaluate distant metastases. Recurrence rate in patients with sentinel lymph node with triple negativity could be considered null because the biological mechanism of local recurrence is different from distant recurrence, which is detected with molecular biology techniques. However, the most relevant reported data were that group III (hematoxylin-eosin-/immuno histochemistry-/molecular biology techniques-) patients have $100 \%$ overall survival. Patients identified as sentinel lymph node positive by molecular biology techniques and negative on routine examination, have a worse prognosis with a larger number of recurrences, metastases, and death. In contrast to patients with negative results by molecular biology techniques have an excellent overall survival. Patients with positive RT-PCR results, with or without positive immunostaining (groups II and I), had a significantly decreased overall survival, when compared with patients with negative results with all the methods. Therefore, this triple negativity could be a positive prognostic factor for overall survival. Although the clinical significance of these techniques is still under investigation, the number of positive cases identified appears to correlate with the number of recurring sentinel lymph node cases negative with routine evaluation. The subset of patients with nested RT-PCR sentinel lymph nodepositive samples would represent a real metastatic deposit. These results reveal the importance of the PCR technique. ${ }^{26}$ Previous studies have raised questions about the meaning of micrometastasis detected by immunohistochemistry or PCR, suggesting that the prognosis does not differ considerably from node-negative disease.$^{27} \mathrm{In}$ a large multicenter, randomized prospective study, the Sunbelt Melanoma Trial found no predictive value of sentinel lymph node RT-PCR analysis. ${ }^{28}$ However, the Sunbelt Melanoma Trial (and other similar studies) 30-month follow-up was a short period to draw a definitive conclusion, despite the larger number of enrolled patients. Our results differ from the results of those studies in the large follow-up patients in group I (hematoxylin-eosin-/immunohistochemistry + /molecular biology techniques + ), and in group II (hematoxylin-eosin-/immunohistochemistry-/molecular biology techniques + ), who did not undergo lymphadenectomies. The survival figure included in our study shows that the more sensitive the technique, the earlier the detection of micrometastases (Figure 1). Therefore, we considered that approximately $10 \%$ of the patients with negative sentinel lymph node examined with routine techniques (hematoxylin-eosin and immunohistochemistry) developed distance metastasis. ${ }^{10}$ The use of more sensitive techniques allows earlier detection of neoplastic cells, which after their multiplication would be detected by less sensitive techniques, such as hematoxylin-eosin or immunohistochemistry. Few patients were enrolled in this study, compared with multicentered studies (Sunbelt Melanoma Trial). However, our population has been selected following strict exclusion criteria, to obtain interesting data. On the other hand, detection effectiveness was assessed using as a gold standard the death or survival of those patients who received no treatment after nested RT-PCR result. Given the length of the follow-up and the number of patients involved, our data suggest the epidemiological impact of sentinel lymph node incomplete study on patients with malignant melanoma stages I and II. If a sentinel lymph node was studied only with hematoxylin-eosin, $25 \%$ of patients would be undertreated because they would fail to receive complete treatment (lymphadenectomy and potential adjuvant treatment). Therefore, these patients would have a higher recurrence rate and a lower overall survival. In case that sentinel lymph nodes were studied with hematoxylin-eosin and immunohistochemistry, the rate of underdiagnosed patients would be $20 \%$. In case sentinel lymph nodes were studied with hematoxylin-eosin, immunohistochemistry, and molecular biology techniques, the solid tumour stratification, which has lymph node dissemination, would be optimized and surgery would be performed only in real metastasized sentinel lymph node patients.

In conclusion, lymph node mapping and sentinel lymph node biopsy seem to be the best choice to determine cutaneous melanoma staging, without previous removal of the lymph node chain. Unnecessary prophylactic lymphadenectomy is avoided, thus reducing morbidity and cost. The joint use of hematoxylin-eosin and immunohistochemistry significantly increases study sensitivity. In addition, more accurate counseling about overall survival prognosis can be offered. The inclusion of molecular biology techniques is of great value for the detection of sentinel node micrometastases in patients with malignant cutaneous melanoma. Thus, staging and treatment of stage I and II patients can be optimized. In our series, those patients who showed negativity with all the three methods had a null recurrence rate. Therefore, this triple negativity could be a positive prognostic factor for overall survival. Thus, even the most insignificant microscopic infiltration of satellite lymph nodes is of utmost importance, both clinically and therapeutically. Given the length of the follow-up and the number of patients involved, study data suggest changes in staging and, therefore, in treatment. 
Regarding molecular biology, this new oncological staging would allow the selection of patients with submicroscopic metastases for a complete treatment (lymphadenectomy and potential adjuvant treatment). On the other hand, several patients with no lymph node metastases should not undergo lymphadenectomies, and overtreatment can be avoided.

\section{Acknowledgements}

We thank Valeria Melia for her assistance with the translation of the manuscript, and Hugo Krupitzki for his methodological counseling.

\section{Disclosure/conflict of interest}

The authors state that they have no conflict of interest to declare.

\section{References}

1 Milton G, Shaw H, McCarthy W. Occult primary malignant melanoma: factors influencing survival. Br J Surg 1977;64:805-808.

2 Hurt M, Santa Cruz D. Tumour of the skin. In: Fletcher C (ed). Diagnostic Histopathology of Tumours, 2nd edn. Churchill Livingtone: Hong Kong, 2000, pp 1357-1472.

3 Matos E, Loria D, Zangarini $N$ (eds). Atlas de Mortalidad Por Cáncer. Argentina 1997-2001. Loria D: Buenos Aires, 2003.

4 AJCC. Carcinoma of the Skin. In: Lippincott Raven Publishers (eds). AJCC Cancer Staging Manual, 6th edn. Springer Verlag: Philadelphia, 2002, pp 201-220.

5 Elder D, Elenitas R. Lesiones pigmentadas benignas y melanoma maligno. In: Elder D, Elenitas R, Jaworsky C, Johnson B(h) (eds). Lever's Histopatología De La Piel, 8th edn. Editorial Inter Médica: Buenos Aires, 1999, pp 549-594.

6 Milton G, Shaw H, McCarthy W, et al. Prophylactic lymph node dissection in clinical stage l cutaneous malignant melanoma: results of surgical treatment in 1319 patients. Br J Surg 1982;69:108-111.

7 Reintgen D. Melanoma nodal metastases: biologic significance and therapeutic considerations. Surg Oncol Clin N Am 1996;5:105-120.

8 Morton D, Wen D, Wong J, et al. Technical details of intraoperative lymphatic mapping for early stage melanoma. Arch Surg 1992;127:392-399.

9 Boi S, Cristofolini P, Togni R, et al. Detection of nodal micrometastases using immunohistochemistry and PCR in melanoma of the arm and trunk. Melanoma Res 2002;12:147-153.

10 Bostick P, Morton D, Turner R, et al. Prognostic significance of occult metastases detected by sentinel lymphadenectomy and reverse transcriptase-polymerase chain reaction in early-stage melanoma patients. J Clin Oncol 1999;17:3238-3244.
11 Blaheta H, Schittek B, Breuninger H, et al. Detection of melanoma micrometastases in sentinel nodes by reverse transcription-polymerase chain reaction correlates with tumor thickness and is predictive of micrometastases disease in lymph node basin. Am J Surg Pathol 1999;23:822-828.

12 Smith B, Selby P, Southgate J, et al. Detection of melanoma cells in peripheral blood by means of reverse transcriptase and polymerase chain reaction. Lancet 1991;338:1227-1229.

13 Reintgen D, Shivers S. Sentinel lymph node micrometástasis from melanoma. Proven methodology and evolving significance. Cancer 1999;86:551-552.

14 Wang X, Heller R, van Voorhis N, et al. Detection of submicroscopic lymph node metastases with polymerase chain reaction in patients with malignant melanoma. Ann Surg 1994;220:768-774.

15 Balch C, Buzaid A, Soong S, et al. New TNM melanoma staging system: linking biology and natural history to clinical outcomes. Semin Surg Oncol 2003;21:43-52.

16 Falco J, Mezzadri N, Montesinos M, (eds). Melanoma. Valor Del Mapeo Linfático En La Cirugía Oncológica. Sigma: Buenos Aires, 2002, pp 72-103.

17 Ioachim $\mathrm{H}$, (ed). Metastatic melanoma. Lymph Node Pathology, 2nd edn. JB Lippincott Company: Philadelphia, 1994, pp 674-681.

18 Messina J, Glass L, Cruse C, et al. Pathologic examination of the sentinel lymph node in malignant melanoma. J Am Surg Pathol 1999;23:686-690.

19 Timar J, Csuka O, Orosz Z, et al. Molecular pathology of tumour metastasis. Pathol Oncol Res 2002;8: 204-219.

20 Macripo G, Quaglino P, Caliendo V, et al. Sentinel lymph node dissection in stage I/II melanoma patients: surgical management and clinical follow-up study. Melanoma Res 2004;14:S9-S12.

21 Cochran A, Wen D, Morton D. Management of the regional lymph nodes in patients with cutaneous malignant melanoma. World J Surg 1992;16:214-221.

22 Ross M. The case for elective lymphadenectomy. Surg Oncol Clin N Am 1992;1:205-222.

23 Shivers S, Wang X, Li W, et al. Molecular staging of malignant melanoma. Correlation with clinical outcome. JAMA 1998;280:1410-1415.

24 Cronin C, Ryan A, Talley E, et al. Tyrosine expression during neuroblast divisions affect later pathfinding by retinal ganglion cells. J Neurosci 2003;23: 11692-11697.

25 Uribe C, Avila M, Zarate M, et al. Melanoma medular amelanótico. MEDUNAB 2002;5:133-135.

26 Denninghoff V, Kahn A, Falco J, et al. Sentinel lymph node: detection of micrometastases of melanoma. A molecular study. Mol Diagn 2005;8:253-258.

27 Scoggins CR, Ross MI, Reintgen DS, et al. Prospective multi-institutional study reverse transcriptase polymerase chain reaction for molecular staging of melanoma. J Clin Oncol 2006;24:2849-2857.

28 Tatlidil C, Parkhill W, Giacomantonio C, et al. Detection of tyrosinase mRNA in the sentinel lymph nodes of melanoma patients is not a predictor short term disease recurrence. Mod Pathol 2007;20:427-434. 